\title{
Research on the Transformation and Upgrading of the "Internet + " Logistics Industry under the New Economic Normal
}

\author{
Ranran Jiang \\ Shandong Xiehe University, Jinan, Shandong, 250107
}

Keywords: New economic normal; Internet +; logistics industry; transformation

\begin{abstract}
The most typical central strategic judgment is the new normal of the economy. This new normal is a trend to a certain extent, and it is not the same as the original stable development. The logistics industry itself is a production-oriented service industry. It has an extremely important basic and strategic role in the national economic construction. Therefore, in recent years, it has received more and more attention from more departments. It is especially important to explore its future development trends to explore new development ideas in the future. Based on this, this paper will focus on the main problems existing in the current logistics industry, and then study the development and changes of the logistics industry under the new economic normal, and finally propose relevant innovative development methods.
\end{abstract}

\section{Introduction}

The advancement of the current scientific and technological revolution has led to the emergence of the Internet economy and the digital economy, especially the integration and development of the Internet and various industries, and the trend has become more and more unstoppable. The logistics industry is the most important industry in the process of our country's economic development. It has a very important position in the national economy and in the Internet, and this status is still rising, and has even become the pillar of our country. Industry. At present, the logistics industry is already in a critical period of transformation. The original logistics industry has been unable to adapt to the current social development needs, so it is necessary to transform in time, make full use of the advantages of the Internet + , realize the sharing of logistics information, and build a new type of Logistics development format to promote the development of the logistics industry.

\section{The problems and causes}

Under the background of the development of the new economic normal, more and higher requirements are put forward for the future development of the logistics industry. The original logistics industry basically has many problems. The existence of these problems restricts and restricts the logistics industry to some extent. The main problem is that the original logistics enterprises are relatively small in scale and distributed, and the functions of logistics enterprises are relatively simple. When comparing regional industries, the capabilities are compared. difference. The main reasons for these problems are the following main aspects.

At present, many localities and departments in our country have fully realized the beneficial effects of the logistics industry on the national economic construction. Therefore, they have basically begun to pay attention to the development of the logistics industry, but in the most fundamentally many logistics enterprises themselves The lack of understanding of the future development prospects of the logistics industry and the fact that the Internet itself can understand the economic benefits brought by the logistics industry are still insufficient [1]. In addition, among the logistics enterprises, many of the relevant business managers are people outside the industry. There are very few talents who really learn the logistics profession. Some people even have not accepted or rarely received relevant knowledge about the logistics industry. Training. This has brought certain limitations and challenges to the further development of the logistics industry, making the industry type of logistics itself limited to warehousing and transportation. The relevant 
management personnel themselves are not aware of the benefits that the advancement and development of Internet technology will bring to the entire logistics industry. It is necessary to reduce the basic cost of circulation and increase certain economic benefits for enterprises. The original management concept and the scarcity of related professionals make the development of logistics enterprises still on a shallow level, focusing only on production, while ignoring the development of logistics itself.

For the logistics industry itself, it mainly needs to use some automated and mechanized equipment to complete the production, but in the actual production process, many logistics companies are afraid that they will lose the relevant sales autonomy, so they will carry out the logistics service. Built, and abandoned the outsourcing [2]. However, the main characteristic of the demand for logistics is that it is generally scattered, and the supply chain of its industry has not yet formed a corresponding scale. Therefore, it can be found that the dispersion of such enterprises and the corresponding logistics services can be found. The single function makes the business sector separate, which makes it difficult for the logistics industry itself to form a certain degree of specialization, resulting in the development level of the entire industry is in a relatively low state for a long time.

At present, the order of the entire market of the logistics industry is relatively confusing. There is a lack of basic integration and planning among the entire market, which makes the standards of equipment and tools used in the logistics process have certain standards. The difference in the speed of logistics leads to a reduction in the cost of logistics [3]. It is precisely because of its reduced logistics speed and rising costs that the development prospects of the entire industry have been limited to some extent. In view of this, if the entire logistics industry wants to improve the economic profit of the enterprise and ensure the development of the whole industry, it must first establish a relatively uniform industry standard, and then fundamentally ensure that the entire logistics industry can develop in a standardized direction.

\section{The characteristics of development}

At present, our country has entered the era of the new normal of economic development. Its main characteristics are that the economic development speed is gradually slowing down, the economic structure has been optimized to a certain extent, the economic development is facing many challenges and the economic development has never been seen before. Power [4]. Among them, the economic growth of medium and high speed is mainly because the economic growth rate has changed from the original high-speed normal mode to the medium-high speed growth mode; the optimization of economic structure is mainly the industrial structure and income distribution structure, demand structure and urban and rural regional structure. The optimization of economic development is mainly due to the gradual transformation of China's economy into innovation; the challenge of economic development is mainly the increasing risk of economic development [5]. Among them, the logistics industry, as the most important pillar of the national economic development, also has a very outstanding performance under the new economic normal.

The logistics industry has already accepted the high-speed growth model of the previous years, both in terms of the growth rate of its total logistics volume and the value of the added value, which is much lower than the original. According to the estimation of relevant professionals, the value added of the logistics industry will remain in a relatively moderate state in the future development period.

At present, the growth rate of steel and coal is still slow for the logistics industry, but the growth rate of consumer goods in the society is rising [6]. This is mainly because the e-commerce logistics industry has proliferated, and the growth trend of express delivery and other industries is still rising. Logistics demand in the western region and rural areas has grown rapidly. It is precisely because of the development of the e-commerce industry that higher requirements are placed on the quality of logistics services.

Due to the development of the Internet, the development of industries driven by innovation has increased. In recent years, the investment rate of the logistics industry has gradually increased, and 
the momentum of investment has become more and more fierce. Therefore, the logistics industry and the financial industry and manufacturing industry There have been certain linkages, and various innovative organizations have been rising and rising, which has led to the formation of a modern logistics system.

On the one hand, the cost of each factor required for logistics development is increasing year by year, and the price that its warehouse needs to pay is rising. In addition, labor costs are also rising, so it is difficult to maintain the industry that originally relied on labor. There is a lack of corresponding professional management talents in the enterprise. On the other hand, the environmental protection costs have increased, and the emissions of transportation vehicles have been limited. It is precisely because of the accumulation of these factors that the logistics industry has been overwhelmed, and the logistics industry has begun to show moderate growth. The problems that were previously ignored have begun to emerge. If the logistics industry wants to achieve long-term development, it must optimize its quality. The benefits are adjusted in a timely manner, relying mainly on innovation to drive future development potential.

\section{The changes have taken place}

In recent years, the Internet economy has continued to develop, and the changes in the logistics industry driven by e-commerce and mobile Internet are also enormous. Mainly because the number of express delivery is rising, the demand for logistics is constantly changing, and the carrier of logistics is no longer limited to the original model. Among them, the increase in the number of express delivery reflects the certain changes in the logistics front-end business; the change in the demand pattern of logistics is mainly reflected in the changes in e-commerce, in which the C2B model of major e-commerce platforms has become the latest growth in logistics demand. Point; logistics in the carrier is no longer limited to high-speed trains, but mainly depends on air cargo; the transformation of the commercialization model mainly depends on the Internet + , resulting in personalized customization.

The development of Internet+ has enabled the logistics industry to find a new way out under such a form. The development of information technology has promoted the logistics industry to improve the logistics speed and industrial integration. In addition, the logistics market has been continuously updated and transmitted. The development of Internet + has changed the original economic development rules and promoted the development of the logistics industry in a smart direction.

\section{The strategy of transformation}

Under the new normal of economic development, make full use of Internet technology, and then focus on integrating thinking and platform thinking, and make full use of the commercial value and economic benefits hidden in the logistics industry. First, you need to take advantage of the appropriate platform. In recent years, due to the rapid rise of logistics platforms in our country, related financial leasing platforms have sprung up, but the business format between platforms has not yet grown. Since the Internet itself has certain openness and sharing, the logistics platform must be innovative on the basis of the Internet.

Second, we must have a certain shared thinking and crowdfunding thinking. The term "crowdfunding" is an Internet concept. It is to concentrate the previously scattered funds so that they can rely on the corresponding resources and information to make money according to the development of the Internet, and finally open up huge in the logistics market. Try the space. Shared thinking is currently the most popular Internet concept, such as sharing bicycles, sharing cars, etc. The emergence of this way of thinking can reduce the idleness of resources and waste of resources in the most fundamental way, and also enable the logistics industry to achieve sharing mode in the future. Bring a certain amount of imagination.

Finally, flow thinking. The original logistics companies mainly obtained certain economic benefits based on sales volume. But now it is in the era of the Internet, so the model of obtaining 
economic benefits is based on big data to carry out corresponding traffic marketing. This kind of sales method is more economical and has the greatest value potential.

Since the development of the Internet has brought certain conditions to the integration between industries, the Internet technology with machinery-related information technology has brought a corresponding driving role for the integration of logistics industry. At present, the development of Internet technology in the logistics industry is deepening, and the linkage between related industries in the logistics industry has been further enhanced, such as financial services and logistics, consulting and logistics, etc. The enhancement of linkage promotes the integration of capital, and thus brings relevant media to the development of the logistics industry. The business of different logistics companies and different industry enterprises will be further expanded, which will bring huge space for the flow of goods, capital and information; in addition, the economic relaxation of relevant regulations and policies The reduction of barriers has brought a huge foundational role in the reconfiguration and integration of industries in the logistics industry. The logistics industry itself is a service-oriented industry, so it is necessary to improve various facilities, and to develop linkages with various industries, and further extend the level of industry and function.

The original logistics industry's marketing model is mainly determined according to the product model, so it is very difficult to transform and upgrade. Due to the development of the Internet, the development of intelligent logistics has been promoted, and a precise marketing model has been born in the logistics industry. In this context, logistics companies should make full use of the convenience brought by big data, optimize the size of their orders, the path and location of logistics, and so on, and then learn about consumer spending habits and location information, and ultimately Guaranteed to bring personalized service to customers. In addition, due to the development of O2O, the services between online and offline can be organically integrated, and it is no longer the original single service. The mobile terminal and PC-side services can be more convenient and high-quality. In this regard, the logistics industry should abandon the active logistics services, so that the informationization degree of the logistics industry can be integrated with the network.

\section{Conclusion}

In general, the logistics industry has ushered in new opportunities for development under the new economic normal, but it still faces certain challenges. In this regard, we should make full use of the advantages of Internet technology, find the integration point between logistics transformation and growth speed, pay attention to the linkage between industries, and pay attention to the needs of customers, so that the quality and efficiency of the logistics industry can be improved accordingly. Under such a situation, it is necessary to accurately grasp the development characteristics of the logistics industry and respond positively. Only in this way can the logistics industry achieve dynamic balance in the process of transformation.

\section{Acknowledgements}

Fund Project: 2017 Jinan Soft Science Research Project

Research project number: 201704051

Research project name: Research on the Development Strategy of Logistics Industry in Jinan City under the Background of "Internet +"

\section{References}

[1] Wang Shanshan. Industrial transformation and upgrading under the new economic normal and "Internet +" [J]. Human, 2016, 199 (4).

[2] Chen Yinghua. Research on the Innovation and Development of Small and Medium-sized Logistics Enterprises under the New Normal[J]. Modernization of Shopping Malls, 2016(11): 47-48. 
[3] Guo Daiwei. Research on the Transformation and Upgrading of Logistics Industry under “Internet +” [J]. Business, 2016(37).

[4] ZHU Tiangao, ZHOU Yanchun. Research on the Transformation and Upgrading Path of Nantong Logistics Enterprise Community under the New Economic Normal[J]. Journal of Nantong Vocational University,2016,30(2):30-32.

[5] Li Xia. Analysis of the development and transformation of the "Internet + " logistics industry under the new normal [J]. China Economic and Trade, 2016 (10): 139-139.

[6] Gao Jiali. Research on the Development Model and Strategy of “Internet +" Logistics Industry under the "Belt and Road” Strategy [J]. Business Economics Research, 2016(24): 91-93. 